MPIKG Public Access

Author Manuscript

Published in final edited form as:

Markushyna, Y., Teutloff, C., Kurpil, B., Cruz, D., Lauermann, I., Zhao, Y., et al. (2019).

Halogenation of aromatic hydrocarbons by halide anion oxidation with

poly(heptazine imide) photocatalyst. Applied Catalysis B: Environmental, 248, 211-

217. doi:10.1016/j.apcatb.2019.02.016.

\title{
Halogenation of Aromatic Hydrocarbons by Halide Anion Oxidation with Poly(heptazine imide) Photocatalyst
}

Yevheniia Markushyna, Christian Teutloff, Bogdan Kurpil, Daniel Cruz, Iver Lauermann, Yubao Zhao, Markus Antonietti and Aleksandr Savateev

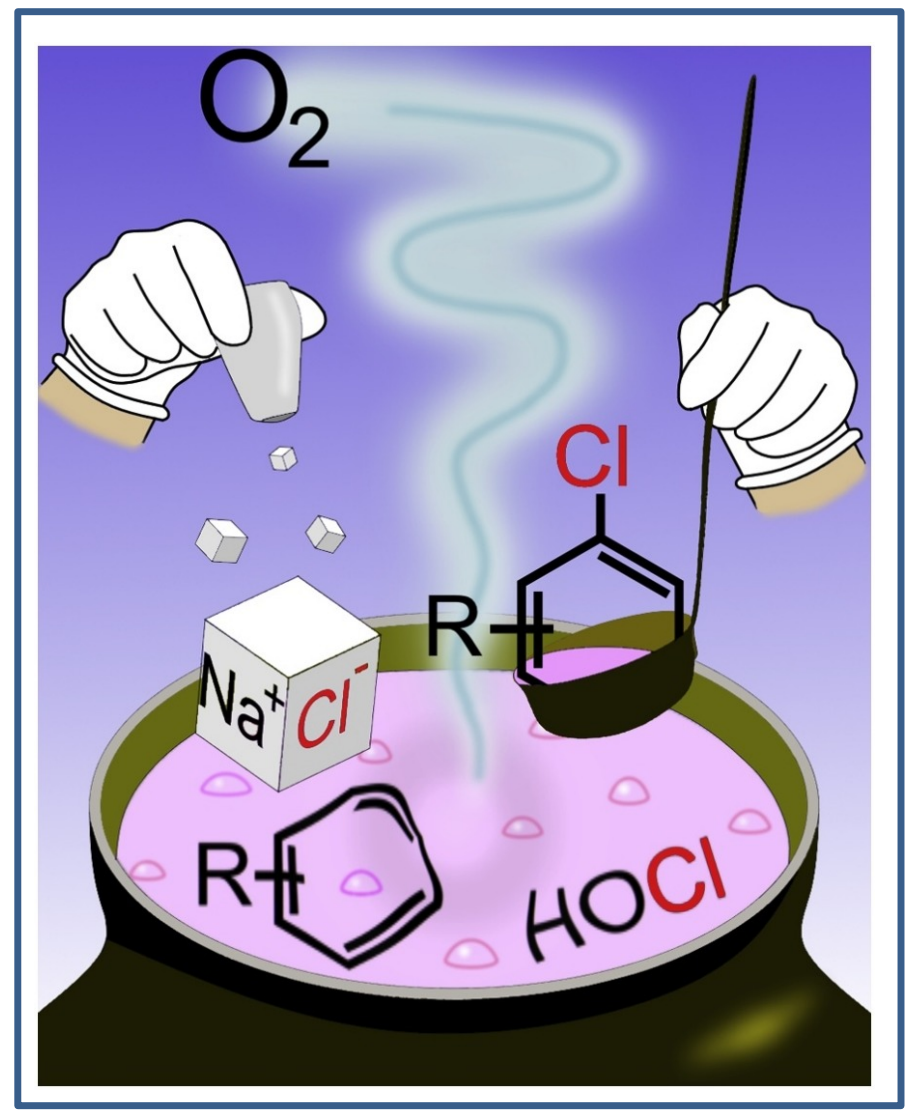

Keywords:

Carbon nitride, Organic photoredox catalysis, Organic synthesis, Chlorination, Photooxidation 


\title{
Halogenation of Aromatic Hydrocarbons by Halide Anion Oxidation with Poly(heptazine imide) Photocatalyst
}

\author{
Yevheniia Markushyna ${ }^{\mathrm{a}}$, Christian Teutloff ${ }^{\mathrm{b}}$, Bogdan Kurpil ${ }^{\mathrm{a}}$, Daniel Cruz, ${ }^{\mathrm{a}}$ Iver Lauermann, ${ }^{\mathrm{c}}$ Yubao \\ Zhao $^{\text {a }}$, Markus Antonietti ${ }^{\mathrm{a}}$, Aleksandr Savateev ${ }^{\mathrm{a}, *}$ \\ ${ }^{a}$ Max-Planck Institute of Colloids and Interfaces, Department of Colloid Chemistry, Research Campus Golm, 14424 \\ Potsdam, Germany \\ ${ }^{\mathrm{b}}$ Freie Universität Berlin, Department of Physics, 14195 Berlin, Germany \\ ${ }^{\mathrm{c}}$ Helmholtz Zentrum Berlin für Materialien und Energie (HZB), 1, Hahn-Meitner-Platz, 14109 Berlin, Germany
}

\begin{abstract}
Halocarbons have tremendous significance for chemical industry. Arylchlorides and arylbromides are typically prepared via Friedel-Crafts reaction, using oxidative halogenation or employing halogen transfer agents, such as $N$-chloro- and $N$-bromosuccineimide. Herein, we show that $\mathrm{K}-\mathrm{PHI}$, a photocatalyst of the carbon nitride family, can easily photooxidize halide anions $\left(\mathrm{Cl}^{-}, \mathrm{Br}^{-}\right)$in aqueous media to promote oxidative halogenation of electron rich aromatic compounds. The technique is convenient, safe and gives the haloaromatic products with excellent yields from non-harmful starting reagents in the most sustainable fashion. We show that solution of $\mathrm{NaCl}$, seawater mimic, can be used as a reagent for photochlorination.
\end{abstract}

Keywords: carbon nitride $\bullet$ organic photoredox catalysis $\bullet$ organic synthesis $\bullet$ chlorination $\bullet$ photooxidation

\section{Introduction}

Chlorinated and brominated hydrocarbons are a pivot of the chemical industry - they are important reagents and solvents. Oxidative halogenation is probably the least harmful method to synthesize halogenated hydrocarbons. [1] According to this approach a halide-anion $\left(\mathrm{Cl}^{-}\right.$or $\left.\mathrm{Br}^{-}\right)$is oxidized in situ by $\mathrm{H}_{2} \mathrm{O}_{2}$ or $\mathrm{S}_{2} \mathrm{O}_{8}{ }^{2-}$ to generate an electrophilic " $\mathrm{Hal}^{++}$" species, which in turn enable electrophilic halogenation of the aromatic substrate. Photocatalytic versions of oxidative chlorination mediated by riboflavin tetraacetate and $\mathrm{Ru}(\mathrm{bpy})_{3} \mathrm{Cl}_{2}$ homogeneous photocatalysts under visible light irradiation were recently reported.[2],[3] These photocatalysts offer rather high conversion of the substrates and good selectivity, but their cost, separation from the product, and their recycling is problematic. In order to overcome existing problems, Liu and Whiting developed a method of chloride anion oxidation to the radical over heterogeneous $\mathrm{Ag} @ \mathrm{AgCl}$ catalyst under visible light irradiation and applied this reaction in chlorination of hydrocarbons.[4],[5] Despite these remarkable advances in the field further development of efficient and sustainable metal free heterogenous photocatalyst is needed.

Polymeric carbon nitride, a visible light heterogeneous photocatalyst, possesses exceptionally high chemical and thermal stability that are a mandatory requirement if a reaction is performed in a highly reactive medium, for example, in the presence of $\mathrm{O}_{2} / \mathrm{O}_{2}{ }^{-{ }^{-}}$couple.[6],[7],[8] Carbon nitrides are tolerant to various organic radicals as it has been shown, for example, in ketalization reaction,[9] styrenes sulfination[10,11] and Diels-Alder cycloaddition.[12] This is to be added to numerous examples of

* Corresponding author

E-Mail address: oleksandr.savatieiev@mpikg.mpg.de 
visible light driven water splitting [13],[14],[15],[16] and $\mathrm{CO}_{2}$ reduction[17] that involve multiple reactive intermediates. Overall the scope of reaction mediated by carbon nitrides is indeed very broad and was recently summarized in reviews.[18],[19] From the usability point of view, as a heterogeneous powder carbon nitrides can be easily separated from the reaction mixture by filtration or centrifugation.

Recently, we also presented an improved modification of polymeric carbon nitride, potassium poly(heptazine imide) (K-PHI), [20-22] which shows a remarkably improved oxidation strength with a potential of valence band $+2.54 \mathrm{~V}$ (Fig. 1). [23]
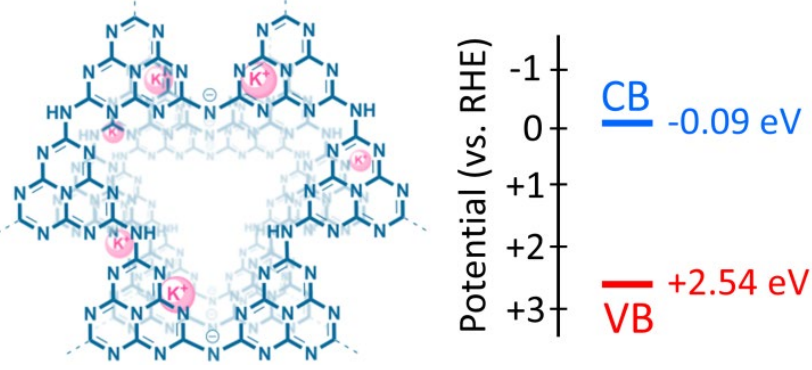

Fig. 1. Chemical structure (left) and band structure (right) of K-PHI.

Carbon nitrides have been already applied in $\mathrm{H}_{2} \mathrm{O}_{2}$ production upon $\mathrm{O}_{2}$ reduction, [24] but the possibility to couple this reaction on the photooxidation side with the oxidative halogenation of the aromatic compounds was not taken into account. This approach is beneficial compared to nonphotocatalytic version $\left(\mathrm{H}_{2} \mathrm{O}_{2} / \mathrm{HCl}\right.$ mixture), because $\mathrm{H}_{2} \mathrm{O}_{2}$ is generated in situ in micro quantities right before consumption (and has not to be added), and is potentially surface-bound and still is in activated form, and therefore substrate over-chlorination or over-oxidation is minimized.[2]

In addition, the use of some carbon nitrides for direct $\mathrm{Cl}^{-}$anion photooxidation was recently demonstrated.[25] If this is performed in water (the natural solvent of simple halide salts), a medium for the selective oxidative chlorination of hydrocarbons is established.[26] Given our recent results on KPHI, which can be applied to challenging reactions like water oxidation,[27] and in organic syntheses to prepare thioamides,[28] 1,3,4-oxadiazoles,[29] disulfides[30], substituted pyridines,[21] and cyclopentanoles[31] we envision this material to be beneficial for even demanding oxidation reactions.

Herein we report photocatalytic oxidation of $\mathrm{Cl}^{-}$and $\mathrm{Br}^{-}$anions by K-PHI in water under aerobic conditions and visible light irradiation. Under these conditions a series of aromatic compounds was converted into their halogenated derivatives with high yields and selectivity.

\section{Experimental}

\subsection{Catalysts preparation}

K-PHI was prepared according to the literature procedure.[21]

$\mathrm{H}-\mathrm{PHI}$ was prepared according to the following procedure. A suspension of K-PHI (408 $\mathrm{mg})$ in $\mathrm{HCl}(18 \mathrm{~mL}, 0.1 \mathrm{M})$ was stirred at room temperature for $24 \mathrm{~h}$. The suspension was subjected to centrifugation (13000 rpm, $10 \mathrm{~min})$. Supernatant layer was decanted and deionized water $(2 \mathrm{~mL})$ was added to the solid. The solid was dispersed by vortex mixer and centrifuged (13000 rpm, $10 \mathrm{~min}$ ) again. Supernatant layer was decanted and washing procedure was repeated in total 7 times until suspension $\mathrm{pH}$ 7. The solid was dried in vacuum overnight.

g-CN was prepared according to the following procedure. Dicyandiamide $(15 \mathrm{~g})$ was heated to $600^{\circ} \mathrm{C}$ with a ramp $2.4^{\circ} \mathrm{C}$ under $\mathrm{N}_{2}$ flow. After cooling to room temperature, solid was finely ground in mortar. 


\subsection{Chlorination of aromatic compounds}

Blue LED $\left(\lambda_{\max }=465 \mathrm{~nm}, 52 \mathrm{~mW} \mathrm{~cm}^{-1}\right)$ was used as a light source in all photocatalytic experiments.

\subsubsection{Method A (König method) for the photocatalytic chlorination}

In a vial a substrate $(0.02 \mathrm{mmol})$, together with $\mathrm{HOAc}(0.2 \mathrm{mmol}, 10$ eq. $)$ and benzylalcohol $(0.12$ mmol, 6 eq. $)$ were dissolved in acetonitrile $(0.5 \mathrm{~mL})$ and $\mathrm{HCl}(0.1 \mathrm{~mL}, 36 \mathrm{wt}$. \% $\mathrm{HCl})$. Then K-PHI $(1 \mathrm{mg})$ was added and the reaction mixture was irradiated under stirring at $30^{\circ} \mathrm{C}$ for $12 \mathrm{~h}$ under $\mathrm{O}_{2}$ atmosphere. After the irradiation $\mathrm{CDCl}_{3}(0.7 \mathrm{~mL})$ and water $(0.1 \mathrm{~mL})$ were added to the reaction mixture. The layers were separated and organic layer was analyzed by ${ }^{1} \mathrm{H}$ NMR using the internal standard $(N, N$ dimethylaniline $(0.01 \mathrm{mmol})$ or anisole $(0.01 \mathrm{mmol})$ in case of $N, N$-dimethylaniline chlorination).

\subsubsection{Method B (pure aqueous conditions, using electron and hole) for the photocatalytic chlorination}

In a vial a substrate $(0.02 \mathrm{mmol})$ was dissolved in acetonitrile $(0.5 \mathrm{~mL}) . \mathrm{HCl}(0.1 \mathrm{~mL}, 36 \mathrm{wt} . \%$, $1.2 \mathrm{mmol})$ and $\mathrm{K}-\mathrm{PHI}(4 \mathrm{mg})$ were added. The reaction mixture was stirred under light irradiation at $30^{\circ} \mathrm{C}$ for $24 \mathrm{~h}$ under $\mathrm{O}_{2}$ atmosphere. After the irradiation $\mathrm{CDCl}_{3}(0.7 \mathrm{~mL})$ and water $(0.1 \mathrm{~mL})$ were added to the reaction mixture. The layers were separated and organic layer was analyzed by ${ }^{1} \mathrm{H}$ NMR using the internal standard $(N, N$-dimethylaniline $(0.01 \mathrm{mmol})$ or anisole $(0.01 \mathrm{mmol})$ in case of $\mathrm{N}, \mathrm{N}$ dimethylaniline chlorination). In the catalytic cyclic experiments K-PHI was washed with acetonitrile (2 times, $2 \mathrm{~mL}$ each), water ( 2 times, $2 \mathrm{~mL}$ each), dried in vacuum overnight and used again.

\subsubsection{General procedure for the photocatalytic bromination}

A vial was charged with a substrate $(0.02 \mathrm{mmol}), \mathrm{K}-\mathrm{PHI}(4 \mathrm{mg}), \mathrm{HBr}(0.1 \mathrm{~mL}, 47 \mathrm{wt} . \%$, $0.9 \mathrm{mmol})$ and acetonitrile $(0.5 \mathrm{~mL})$. A suspension was irradiated at vigorous stirring at $30^{\circ} \mathrm{C}$ for $24 \mathrm{~h}$ under $\mathrm{O}_{2}$ atmosphere. After the irradiation $\mathrm{CDCl}_{3}(0.7 \mathrm{~mL})$ and water $(0.1 \mathrm{~mL})$ were added to the reaction mixture. The layers were separated and organic layer was analyzed by ${ }^{1} \mathrm{H}$ NMR or GC-MS using the internal standard $(N, N$-dimethylaniline $(0.01 \mathrm{mmol})$ or anisole $(0.01 \mathrm{mmol})$ in case of $N, N$ dimethylaniline chlorination).

\subsubsection{Procedure of K-PHI regeneration}

K-PHI after the reaction was separated by centrifugation, washed with acetonitrile ( 2 times, $2 \mathrm{~mL}$ each) and with water (2 times, $2 \mathrm{~mL}$ each). $\mathrm{KOH}$ solution $(9 \mathrm{~mL}, 0.1 \mathrm{M})$ was added to the catalyst and suspension was stirred for $20 \mathrm{~h}$. The catalyst was separated by centrifugation, washed with water ( 3 times, $3 \mathrm{~mL}$ each), once with methanol and dried in vacuum overnight.

\subsubsection{Method of the photocatalytic chlorination with $\mathrm{NaCl}$}

In a vial a substrate $(0.06 \mathrm{mmol})$ was dissolved in acetonitrile $(5 \mathrm{~mL}) . \mathrm{HCl}(20 \mu \mathrm{L}, 36 \mathrm{wt} . \%)$, $\mathrm{NaCl}(48.8 \mathrm{mg})$, water $(1.4 \mathrm{~mL})$ and K-PHI $(5 \mathrm{mg})$ were added. The reaction mixture gave two liquid phases - organic and aqueous. The reaction mixture was vigorously stirred under light irradiation at $30^{\circ} \mathrm{C}$ for $24 \mathrm{~h}$ under $\mathrm{O}_{2}$ atmosphere. After the irradiation organic phase was separated and acetonitrile was 
distilled off, but some water left. $\mathrm{CDCl}_{3}(0.5 \mathrm{~mL})$ was added to the residual water, layers were separated and organic layer was analyzed by ${ }^{1} \mathrm{H}$ NMR using the internal standard (dimethylaniline (0.01 mmol)).

\subsubsection{Oxidative chlorination of anisole using a mixture of $\mathrm{HCl}$ and $\mathrm{H}_{2} \mathrm{O}_{2}$}

A mixture of anisole (0.02 mmol), $\mathrm{HCl}(0.1 \mathrm{~mL}, 36 \mathrm{wt} \%$, $1.2 \mathrm{mmol}), \mathrm{H}_{2} \mathrm{O}_{2}(80 \mu \mathrm{L}, 30 \mathrm{wt} . \%, 0.08 \mathrm{mmol})$ in acetonitrile $(0.5 \mathrm{~mL})$ was stirred at $30^{\circ} \mathrm{C}$ for $24 \mathrm{~h}$ in dark. $\mathrm{CDCl}_{3}(0.7 \mathrm{~mL})$ was added to the reaction mixture, organic phase was separated, dried over anhydrous $\mathrm{Na}_{2} \mathrm{SO}_{4}$ and analyzed by ${ }^{1} \mathrm{H} \mathrm{NMR}$ using $N, N$ dimethylaniline $(0.01 \mathrm{mmol})$ as internal standard.

\subsection{Characterization}

${ }^{1} \mathrm{H}$ NMR spectra were recorded on Agilent $400 \mathrm{MHz}$ spectrometer using signal of $\mathrm{CDCl}_{3}(7.26$ ppm) as a reference. Agilent 6890 Network GC System coupled with Agilent 5975 Inert Mass Selective detector (electron ionization) were used to further analyze the composition of the reaction mixture and to obtain mass spectra of the products. Conversion and selectivity of aromatic compounds chlorination were calculated from ${ }^{1} \mathrm{H}$ NMR spectra. Exemplary NMR spectrum of anisole chlorination is shown in Fig. S1, N,N-dimethylaniline - Fig. S2, 1,3-dimethoxybenzene - Fig. S3.

EPR spectra at X-band $(9 \mathrm{GHz})$ and room temperature were recorded on a lab-built spectrometer comprised of an ER041 MR microwave bridge with 4122 SHQE probe head, AEG electromagnet controlled by ER081S/BH15 power supply/controller (Bruker Biospin, Karlsruhe, Germany), a SR810 lock-in amplifier (Stanford Research Systems, USA), and a 53181A frequency counter (Agilent Technologies, USA). N@C60 was used as g-standard for magnetic field calibration.[32] The XPS measurements were performed using CISSY equipment in ultra-high vacuum (UHV), with a SPECS XR $50 \mathrm{X}$-Ray gun using Mg Ka radiation and Combined Lens Analyser Module (CLAM). Energy-dispersive X-ray (EDX) investigations were conducted on a Link ISIS-300 system (Oxford Microanalysis Group) equipped with a $\mathrm{Si}(\mathrm{Li})$ detector and an energy resolution of $133 \mathrm{eV}$.

\section{Results and discussion}

\subsection{Reaction conditions of photocatalytic chlorination of anisole}

Hering and König have shown that a multicomponent system including riboflavin tetraacetate (RFT) as a homogeneous photocatalyst, $\mathrm{HCl}, \mathrm{O}_{2}, 4$-methylbenzylalcohol and acetic acid, can be used to run oxidative chlorination of aromatic compounds under irradiation with $\lambda=455 \mathrm{~nm}$.[2] We simply used these conditions as a starting point in our investigation, except that RFT was replaced by the heterogeneous K-PHI photocatalyst. Indeed, we observed quantitative conversion of anisole along with excellent selectivity when benzylalcohol was used as electron/proton donor (Table 1, entry 1).

The effect of temperature on the $p$-chloroanisole yield was also studied. In the presence of benzyl alcohol and acetic acid higher yield was obtained at higher temperature (Table A.1). On the other hand, using isopropanol as electron donor without acetic acid the dependence was opposite - lower yield of chloroanisoles was observed at higher temperature (Table A.4). These results could be explained as follows. Benzyl alcohol gives more stable radical upon oxidation then isopropyl alcohol. Furthermore, under the König's conditions acetic acid is converted into peracetic acid, the oxidazing agent, that is more stable at elevated temperature compared with $\mathrm{H}_{2} \mathrm{O}_{2}$ produced under the simplified conditions. Having these results further experiments were performed at $30^{\circ} \mathrm{C}$, the optimal temperature for this process. 
We have also tested other common alcohols for the König conditions - EtOH and $\mathrm{MeOH}$. In these cases, conversion of anisole was lower (Table A.2), which can be explained by the lower stability of the intermediary radical cation formed during the alcohol oxidation. However, the choice of ${ }^{i} \mathrm{PrOH}$ can be justified as an environmentally friendly reagent as the product of its oxidation is acetone.

Because of highly positive valence band potential of $\mathrm{K}-\mathrm{PHI},+2.54 \mathrm{~V}$ vs $\mathrm{RHE}, \mathrm{Cl}^{-}$theoretically can be oxidized to " $\mathrm{Cl}^{+}$" via a two electron process $\left(E^{0}=+1.36 \mathrm{~V}\right)$ by the photocatalyst without using auxiliary reagents - acetic acid and alcohol, i.e. in this desired case we really take the "naked" catalyst and use both photogenerated electron and hole for the reaction. Under these simplified conditions, we were pleased to observe $65 \%$ conversion of anisole along with high selectivity after only $12 \mathrm{~h}$ (entry 2 ). When the amount of K-PHI was increased to $4 \mathrm{mg}$ and time of irradiation to $24 \mathrm{~h}$, quantitative conversion of anisole was obtained (entry 3 ).

Table 1

Reaction conditions optimization of anisole chlorination.

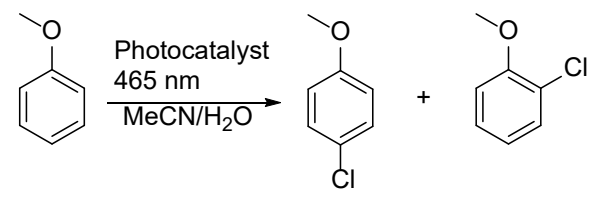

\begin{tabular}{|c|c|c|c|c|c|}
\hline Entry & Photocatalyst & Alcohol & Time, $\mathrm{h}$ & Conversion, ${ }^{[\mathrm{b}]} \%$ & Yield, ${ }^{[c]} \%$ \\
\hline $1^{[\mathrm{a}]}$ & K-PHI (1 mg) & $\mathrm{BnOH}$ & 12 & 100 & $98(1: 0.31)$ \\
\hline $2^{[\mathrm{d}]}$ & K-PHI (1 mg) & - & 12 & 65 & $64(1: 0.32)$ \\
\hline $3^{[\mathrm{d}, \mathrm{e}]}$ & K-PHI (4 mg) & - & 24 & 100 & $99(1: 0.31)$ \\
\hline $4^{[\mathrm{d}, \mathrm{f}]}$ & K-PHI (4 mg) & - & 24 & 78 & $75(1: 0.31)$ \\
\hline $5^{[\mathrm{d}, \mathrm{g}]}$ & K-PHI (4 mg) & - & 24 & 61 & $53(1: 0.33)$ \\
\hline $6^{[\mathrm{d}, \mathrm{h}]}$ & K-PHI (4 mg) & - & 24 & 39 & $35(1: 0.35)$ \\
\hline $7^{[\mathrm{d}, \mathrm{i}]}$ & K-PHI (4 mg) & - & 24 & 100 & $99(1: 0.34)$ \\
\hline $8^{[\mathrm{d}, \mathrm{j}]}$ & K-PHI (1 mg) & - & 13 & 28 & $26(1: 0.41)$ \\
\hline $9^{[\mathrm{k}]}$ & Na-PHI(4 mg) & - & 24 & 39 & $\begin{array}{l}35 \\
(1: 0.32)\end{array}$ \\
\hline $10^{[\mathrm{k}]}$ & H-PHI & - & 24 & 46 & $\begin{array}{l}43 \\
(1: 0.33)\end{array}$ \\
\hline $11^{[\mathrm{k}]}$ & $\mathrm{g}-\mathrm{CN}$ & - & 24 & 44 & $\begin{array}{l}42 \\
(1: 0.29)\end{array}$ \\
\hline $12^{[\mathrm{k}]}$ & $\mathrm{Ru}(\mathrm{bpy}){ }_{3} \mathrm{Cl}_{2}$ & - & 24 & 100 & $\begin{array}{l}97 \\
(1: 0.22)^{[\mathrm{h}]}\end{array}$ \\
\hline $13^{[\mathrm{k}]}$ & $\operatorname{Ir}(p p y)_{3}$ & - & 24 & 0 & 0 \\
\hline $14^{[\mathrm{k}]}$ & RFT & - & 24 & 0 & 0 \\
\hline
\end{tabular}

[a] anisole $0.02 \mathrm{mmol}$; $\mathrm{HCl}$ (36 wt. \%) $0.1 \mathrm{~mL}$; HOAc $0.2 \mathrm{mmol}$; alcohol $0.12 \mathrm{mmol}$; $\mathrm{MeCN} 0.5 \mathrm{~mL}$; $\mathrm{T}=30^{\circ} \mathrm{C}$; electron scavenger $-\mathrm{O}_{2}$; LED module $465 \mathrm{~nm}$. [b] determined by ${ }^{1} \mathrm{H}$ HMR using $N, N$-dimethylaniline as an internal standard. [c] total yield of $o$ - and $p$-chloroanisole. The ratio between $p$ - and $o$-chloroanisole was determined from ${ }^{1} \mathrm{H}$ NMR spectra and is given 
in parentheses. [d] anisole $0.02 \mathrm{mmol} ; \mathrm{HCl}\left(36\right.$ wt. \%) $0.1 \mathrm{~mL}$; MeCN $0.5 \mathrm{~mL} ; \mathrm{T}=30^{\circ} \mathrm{C}$; electron scavenger $-\mathrm{O}_{2}$; LED module $465 \mathrm{~nm}$. [e] first run; [f] second run; [g] third run; [h] fourth run; [i] K-PHI was recovered and washed with $\mathrm{KOH}$; [j] triethylammonium chloride (TEACl) $(0.2 \mathrm{mmol})$ was used as a source of $\mathrm{Cl}^{-} ; \mathrm{H}_{2} \mathrm{SO}_{4}(0.2 \mathrm{mmol})$; [k] anisole $0.02 \mathrm{mmol}$; catalyst $4 \mathrm{mg}$; $\mathrm{HCl}\left(36\right.$ wt. \%) $0.1 \mathrm{~mL}$; MeCN $0.5 \mathrm{~mL} ; \mathrm{T}=30^{\circ} \mathrm{C}$; electron scavenger $-\mathrm{O}_{2}$; LED module $465 \mathrm{~nm}$. The ratio between $d i$ - and $p$-chloroanisole was determined from ${ }^{1} \mathrm{H}$ NMR spectra and is given in parentheses.

After few cycles of using the same catalyst we observed essential drop of activity of K-PHI. (entries 4-6). The result can be explained by highly acidic reaction conditions that lead to substitution of potassium by hydrogen. It is in accord with the entry 10, where we studied H-PHI photocatalyst prepared from K-PHI by treatment with $\mathrm{HCl}$. Furthermore our hypothesis was supported by Energy Dispersive XRay Analysis (EDX). Potassium content in fresh K-PHI was $10.44 \mathrm{w} \%$, but after the photocatalytic reaction it decreased to $0.11 \mathrm{w} \%$. Nevertheless, potassium can be easily and quickly returned into K-PHI structure by treatment of the spent catalyst with $\mathrm{KOH}$. As suggested by the EDX analysis potassium content in the regenerated sample was $10.07 \mathrm{wt} . \%$, while X-Ray diffraction pattern and FT-IR spectrum of the regenerated sample and the starting material were identical. The regenerated K-PHI gave the same activity as the fresh material (entry 7). The experimental data unambiguously indicate the correlation between potassium content in the photocatalyst and its activity in the oxidative chlorination of anisole and may be explained as follows. Potassium cations in the structure of K-PHI compensate negative charge localized at nitrogen atoms. Making comparison with the structures of $N$-chloro compounds, e.g. $N$ chlorosuccineimide, chloranmine- $\mathrm{T}$ etc., negatively charged $\mathrm{N}$-atoms are essential for chlorine coordination by K-PHI. At the same time, coordination of $\mathrm{Cl}$ atoms by covalent carbon nitrides, i.e. g$\mathrm{CN}$ or H-PHI, is also possible, but obviously less efficient. Therefore covalent carbon nitrides are significantly less active in the chlorination reaction.

Under water-free conditions and using triethylammonium chloride (TEACl) as a $\mathrm{Cl}^{-}$source, anisole gave products of chlorination (entry 8), too. Nevertheless, an acidic medium and a solvent stabilized $\mathrm{Cl}^{+}$are essential to close the reaction path as explained in Fig. 3. The blank experiments have proven that without light and $\mathrm{O}_{2}$ no anisole chlorination takes place (Table A.3).

For comparison purposes, different forms of carbon nitrides were studied. Thus, Na-PHI[21] gave chloroanisoles in 35\% yield (entry 9). H-PHI[22] produced chloroanisoles with $43 \%$ yield (entry 10). Similarly graphitic carbon nitride ( $\mathrm{g}-\mathrm{CN}$ ) gave chloroanisoles with $42 \%$ yield (entry 11 ). Among the investigated homogenous catalysts, only $\mathrm{Ru}(\mathrm{bpy})_{3}$ works in this reaction (enrty 12 ), but recovery of this photocatalyst is still challenging. RFT, without acetic acid and alcohol, did not furnish any chloroanisoles (entry 14).

In order to evaluate the effect of $\mathrm{H}_{2} \mathrm{O}_{2}$ concentration on the reaction of anisole chlorination we used a mixture of $\mathrm{H}_{2} \mathrm{O}_{2}$ and $\mathrm{HCl}$. In this case conversion of anisole was $100 \%$, while selectivity of $p$ chloroanisole was only $6 \%$. The main products were dichloroanisole $(41 \%)$ and trichloroanisole $(51 \%)$ chloroanisoles. These results underline the advantage of the photocatalytic approach versus nonphotocatalytic $-\mathrm{H}_{2} \mathrm{O}_{2}$ is generated in micro quantities right prior reaction and therefore high selectivity toward monochlorinated hydrocarbons is achieved.

The structure of the photocatalyst before and after the photocatalytic reaction was investigated by EDX and X-Ray Photoelectron spectroscopy (XPS) (Fig. 2). The results suggest that under the reaction conditions surface of K-PHI underwent some chemical changes. Thus, XPS analysis after the reaction the surface of the photocatalyst has partially hydrolyzed as suggested by the appearance of the peak at $291.2 \mathrm{eV}$ in the $\mathrm{C} 1 \mathrm{~s}$ spectrum, which is related to the surface $\mathrm{C}=\mathrm{O}$ bonds. Similarly, in N 1s spectrum a fraction of $\mathrm{NH}_{\mathrm{x}}$ groups observed as a peak at $400.4 \mathrm{eV}$ increased.. We propose that these changes are a result of partial hydrolysis of K-PHI, in particular $\mathrm{C}-\mathrm{N}=\mathrm{C}$ bonds. Furthermore, EDX analysis revealed that $\mathrm{Cl}$ content in the catalyst increased from $0.7 \mathrm{wt}$. $\%$ to $2.31 \mathrm{wt} . \%$ after the reaction. Presence of $\mathrm{Cl}$ in 
K-PHI after the reaction was also confirmed by XPS analysis (Fig. A.7). Typically chlorine bound to carbon atom appears above $200 \mathrm{eV}$, while peaks at $197.2 \mathrm{eV}$ and $198.7 \mathrm{eV}$ in $\mathrm{Cl} 2 \mathrm{p}$ spectrum are apparently derived from inorganic chlorine, e.g. Cl-.[33] All in all, the structure of K-PHI was subjected to partial hydrolysis during the photocatalytic chlorination. Nevertheless, these changes are not critical for the photocatalytic activity as can be concluded from the recycling and regeneration experiments (Table $1)$.
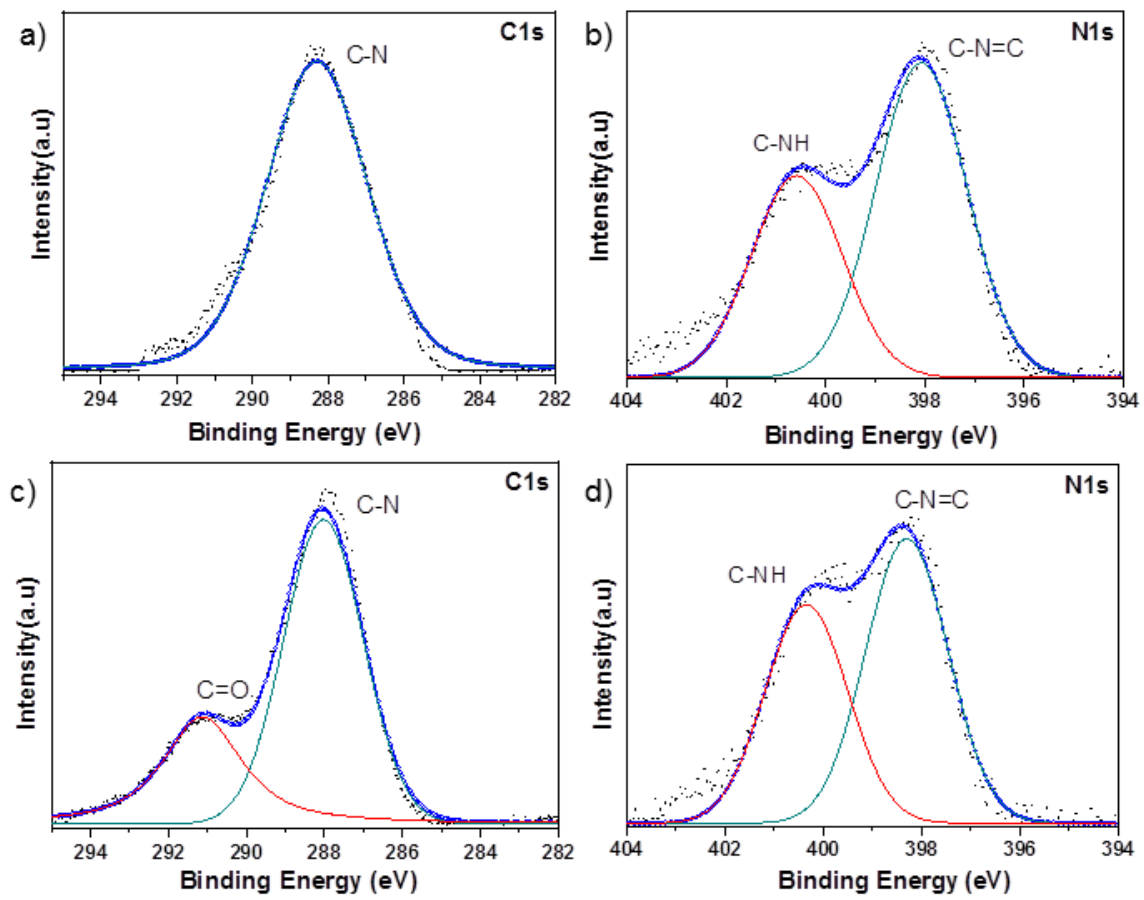

Fig. 2. X-Ray Photoelectron spectroscopy (XPS): a) C 1s spectrum of fresh K-PHI, b) N 1 s spectrum of fresh K-PHI, c) C 1s spectrum of K-PHI after the photochlorination reaction, d) N 1s spectrum of K-PHI after the photochlorination reaction.

\subsection{Oxidative halogenation of aromatic hydrocarbons catalysed by K-PHI}

This very practical approach, using aqueous $\mathrm{HCl}$ and $\mathrm{O}_{2}$, as the only reagents was further extended to chlorinate electron rich aromatic compounds. A series of chlorinated benzenes were synthesized under the similar conditions (Scheme 1).

Scheme 1. Chlorination of aromatic substrates catalyzed by K-PHI 
<smiles>COc1cccc(Cl)c1</smiles>

$99(100)^{[a]}$ $99(100)^{[b]}$ [1:0.31] $]^{[c]}$<smiles>COc1ccc(Cl)c(OC)c1</smiles>

55 (55)[a] $34(36)^{[b]}$<smiles>COc1ccc(Cl)cc1Cl</smiles><smiles>COc1ccc(Cl)cc1Cl</smiles><smiles>CN(C)c1cccc(Cl)c1</smiles>

$$
99(100)^{[a]}
$$

40 (44)[a]

$34(36)^{[b]}$

$99(100)^{[b]}$<smiles>CC(=O)Nc1cccc(Cl)c1</smiles>

$$
\begin{aligned}
& 66(67)^{[a]} \\
& 98(100)^{[b]}
\end{aligned}
$$$$
\text { [1:0.1 }]^{\text {[e] }}
$$<smiles>COc1cc(OC)c(Cl)c(OC)c1</smiles><smiles>COc1c(Cl)cc(Cl)c(OC)c1OC</smiles>

99 (100)[a]

[0.2:1 $]^{[g]}$

[a] Substrate $0.02 \mathrm{mmol}$; $\mathrm{HCl}\left(36\right.$ wt. \%) $0.1 \mathrm{~mL}$; MeCN $0.5 \mathrm{~mL}$; K-PHI (4 mg); electron scavenger $\mathrm{O}_{2}$; LED module $465 \mathrm{~nm}$; temperature $30^{\circ} \mathrm{C} ; 24 \mathrm{~h}$. Conversion (given in parentheses) and yield were determined by ${ }^{1} \mathrm{H}$ NMR using $N, N-$ dimethylaniline or anisole (in case of $N, N$-dimethylaniline) as an internal standard. [b] The values obtained when chlorination was performed according to the method A (see ESI) using alcohol as electron donor. [c] The ratio between $p$ - and $o$ chloroanisole. [d] The ratio between $p$ - and $o$-chloro-N,N-dimethylaniline. [e] The ratio between $N$-(4-chlorophenyl)acetamide and $N$-(2-chlorophenyl)acetamide; [f] The ratio between mono- and dichloro-1,3,5-trimethoxybenzene; [g] The ratio between di- and trichloro-1,2,3-trimethoxybenzene.

The reaction went smoothly with the substrates bearing strong EDG (electron donating group). With such substrates conversion and yield were almost quantitative. In case of moderate EDG (acetanilide) the yield and conversion were moderate. The reaction with weak EDG did not proceed or led to chlorination of the side chain.

The proposed approach of halide anion oxidation was also applied in oxidative bromination reaction under the photocatalytic conditions using aqueous $\mathrm{HBr}$ as $\mathrm{Br}^{-}$source and $\mathrm{H}^{+}$donor. The results are summarized in Scheme 2.

Bromination as well as chlorination worked well with the substrates with strong electron donating groups (EDG), such as -OMe, $-\mathrm{NMe}_{2}$, and led to the quantitative conversion and yield. In case of cresols, weak EDG substituents, the reaction occurred not only in the aromatic core, but gave also substantial amount of side-chain brominated products. Different behavior of cresols compared with the other substrates is explained by the ability of these molecules to give relatively stable benzyl radicals. Therefore side chain bromination readily proceeds via radical substitution along with electrophilic substitution at the aromatic ring. 
Photocatalyst

$465 \mathrm{~nm}$

$\overrightarrow{\mathrm{HBr}, \mathrm{MeCN} / \mathrm{H}_{2} \mathrm{O}}$<smiles>Br[R]1ccc(Br)cc1</smiles><smiles>COc1ccc(Br)cc1</smiles>

$99(100)$<smiles>COc1cc(OC)c(Br)c(OC)c1Br</smiles>

$95(100)$<smiles>COc1ccc(Br)cc1Cl</smiles>

$11(12)$<smiles>COc1ccc(Br)c(OC)c1OC</smiles><smiles>Cc1ccc(C)c(Br)c1</smiles>

$$
\begin{array}{ll}
99(100) & 75(75) \\
{[1: 0.2]^{[b]}} & {[0.36: 1]^{[c]}}
\end{array}
$$<smiles>COc1ccc(Br)c(OC)c1</smiles>

$98(100)$<smiles>Cc1ccc(CBr)cc1</smiles><smiles>Cc1ccc(Br)c(C)c1</smiles>

57 (95)

[a] Substrate $0.02 \mathrm{mmol}$; $\mathrm{HBr}$ (48 wt. \%) $0.1 \mathrm{~mL}$; MeCN $0.5 \mathrm{~mL}$; K-PHI (4 mg); electron scavenger $\mathrm{O}_{2}$; LED module $465 \mathrm{~nm}$; temperature $30^{\circ} \mathrm{C} ; 24 \mathrm{~h}$. Conversion (given in parentheses) and yield were determined by ${ }^{1} \mathrm{H}$ NMR using $N, N-$ dimethylaniline or anisole (in case of N,N-dimethylaniline) as an internal standard. [b] The ratio between mono- and dibromo1,2,3-trimethoxybenzene is given in brackets. [c] The ratio between aryl- and alkyl- substituted $p$-cresol is given in brackets.

\subsection{Mechanism of the oxidative photohalogenation reaction with $K-P H I$}

The mechanism of the oxidative halogenation photocatalyzed by K-PHI is sketched in Fig. 3. Upon excitation with visible light a bound hole-electron pair is formed. At the conductive band site dioxygen, being an electron scavenger is reduced to $\mathrm{H}_{2} \mathrm{O}_{2}$. As evidenced by the EPR spectrum on Fig. 3a the intensity of the signal related to the uncoupled electron in the catalyst in the presence of the hole scavenger (benzyl amine) under irradiation is much intense and sharper compared to the EPR spectrum in dark.[34] This is explained by the formation of the radical anion of K-PHI in the presence of electron donor. When all components of the chlorination reaction were mixed together, under the irradiation and in the dark there is no significant difference between the EPR spectra, proving that in our case $\mathrm{O}_{2}$ from the air acts as electron scavenger, while $\mathrm{Cl}^{-}$acts as an electron donor. The formation of $\mathrm{H}_{2} \mathrm{O}_{2}$ was confirmed by its colored complex with Ti(IV), which gave the additional absorption at $400 \mathrm{~nm}$ (Fig. 3b). The ultimate product of $\mathrm{O}_{2}$ reduction, hydrogen peroxide, reacts readily with the large excess of water and hydrochloric acid giving rise to the active electrophilic species "HOCl" as the chlorinating agent.[35] This "HOCl" subsequently reacts with anisole in an electrophilic aromatic substitution reaction. At the valence band site oxidation of $\mathrm{Cl}^{-}$to $\mathrm{Cl}^{+}$via a two-electron process takes place by the photogenerated holes. The chloride anion was previously reported as a hole scavenger for both $\mathrm{TiO}_{2}$ and carbon nitride as the photocatalysts.[36],[25]. Quenching of the potentially surface bound $\mathrm{Cl}^{+}$by water under liberation of a $\mathrm{H}^{+}$leads to the active electrophilic "HOCl" species. The presence of active chlorine was detected by Quantofix Chlorine test strips (Fig. 3d). The exact structure of the active species hiding electronically the $\mathrm{Cl}^{+}$remains under debate, and herein, for the convenience of mechanism explanation, we denoted it as "HOCl". 
a)

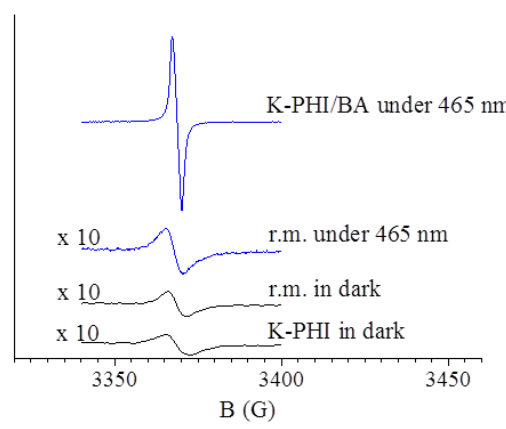

d)

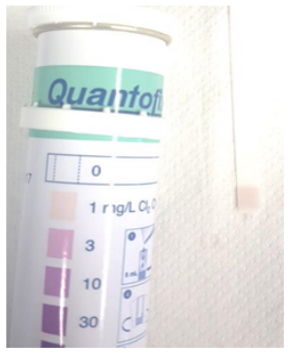

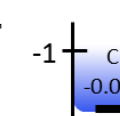
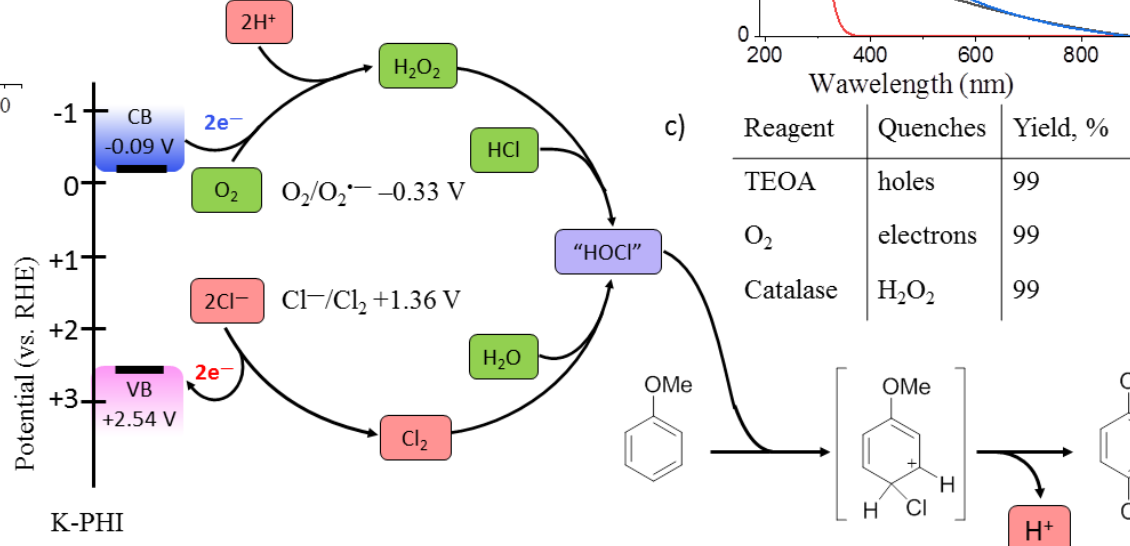

b)

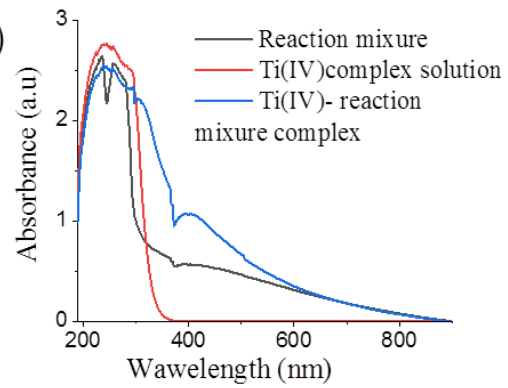

c)

\begin{tabular}{l|l|l} 
Reagent & Quenches & Yield, \% \\
\hline TEOA & holes & 99 \\
$\mathrm{O}_{2}$ & electrons & 99
\end{tabular}

Catalase

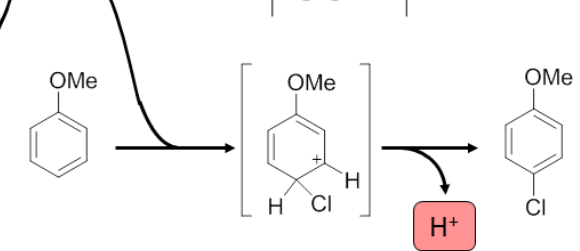

Fig. 3. A proposed mechanism of electrophilic halogenation via $\mathrm{Hal}^{-}$photooxidation and $\mathrm{O}_{2}$ reduction, both resulting in the reactive "HOCl" species. Anisole is given as an example. Conduction and valence band potentials of K-PHI were adapted from the literature.[23] a) EPR spectra of the reaction mixture (anisole $0.02 \mathrm{mmol}, \mathrm{HCl}, \mathrm{MeCN}$ ) in dark and under irradiation $(465 \mathrm{~nm})$ magnified 10 times; EPR spectrum of K-PHI in the presence of hole scavenger (benzylamine) under light irradiation $(465 \mathrm{~nm}) \mathrm{b}) \mathrm{UV}$-vis spectra of the reaction mixture and its complex with Ti(IV) d) active chlorine detection using Quantofix Chlorine test strip. c) results of the reactions with different quenchers.

To prove that formation of "HOCl" occurs via two different pathways we carried out the reaction in the presence of few quenchers (Fig. 3c). As a result TEOA (hole scavenger) and catalase $\left(\mathrm{H}_{2} \mathrm{O}_{2}\right.$ scavenger) did not affect the product yield, which means that formation of $\mathrm{HOCl}$ proceeds by two different ways independently: (1) from $\mathrm{H}_{2} \mathrm{O}_{2}$ and $\mathrm{HCl}$ and (2) quenching of $\mathrm{Cl}_{2}$ with $\mathrm{H}_{2} \mathrm{O}$.

An interesting characteristic of K-PHI is that it is a capacious "electron buffer" to decouple the oxidative and reductive electron fluxes and kinetics, as shown recently.[30] This effect certainly improves its activity in anisole oxidative chlorination, while classical polymeric mpg-CN did not demonstrate significant activity in the described reactions.

In agreement with the proposed mechanism is its high selectivity - only mono chlorinated products were obtained. This implies that the process is electrophilic rather than radical. Moreover, conversion and selectivity observed with both the original König's method and without hole scavenger (benzyl alcohol) are the same, which proves that the reaction proceeds via formation of the same electrophilic agent. Interesting, when dry 1,4-dioxane saturated with $\mathrm{HCl}$ was used as solvent and the $\mathrm{Cl}^{-}$source, 1,4-dioxane polychlorination along with chloroanisole were detected (Fig. A.4). This experiment suggests that the presence of water is important to quench $\mathrm{Cl}^{+}$in situ to give the electrophilic "HOCl" species rather than reacting with $\mathrm{Cl}^{-}$to give $\mathrm{Cl}_{2}$, the first entering the electrophilic substitution reaction rather than the radical chlorination reaction typical for $\mathrm{Cl}_{2}$ under light.

Given that $97 \%$ of all water on Earth is sea water with sodium chloride as a major solute, we have used for curiosity this abundant resource (a $35 \mathrm{~g} \cdot \mathrm{L}^{-1}$ solution of $\mathrm{NaCl}$ in water) to run photocatalytic oxidative chlorination of anisole, and chloroanisoles were indeed formed (Fig. A.5). Using the experimental procedure described above (paragraph 2.2.5) we achieved 62\% conversion of anisole and $51 \%$ yield using $\mathrm{NaCl}$ solution as a source of chlorine. In accord with the results above, in this case 
halogenation worked well with the substrates bearing strong electron donating group too (Scheme 3). Note that here the yields were not optimized, e.g. by $\mathrm{pH}$ variation, but we only wanted to illustrate the principal possibilities.

Scheme 3. Chlorination of aromatic substrates catalyzed by K-PHI with $\mathrm{NaCl}^{[\mathrm{a}]}$

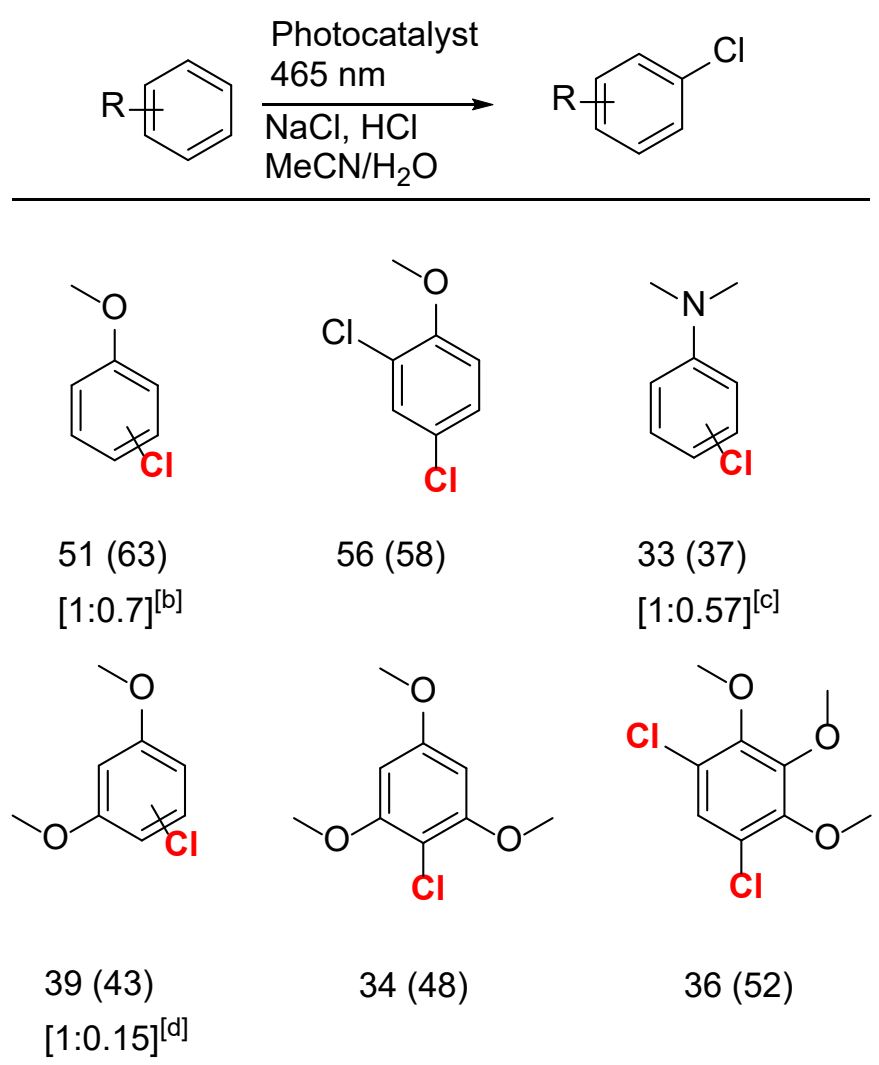

[a] Substrate 0.06 mmol; $\mathrm{HCl}$ (36 wt. \%) $20 \mu \mathrm{L}$; MeCN 5 mL; K-PHI (5 mg); electron scavenger $\mathrm{O}_{2}$; LED module $465 \mathrm{~nm} ; \mathrm{T}=30^{\circ} \mathrm{C} ; 24 \mathrm{~h}$. Conversion (given in parentheses) and yield were determined by ${ }^{1} \mathrm{H}$ NMR using $N, N$-dimethylaniline or anisole (in case of $N, N$-dimethylaniline) as an internal standard. [b] The ratio between $p$ - and $o$-chloroanisole. [c] The ratio between $p$ - and $o$-chloro- $N, N$-dimethylaniline. [d] The ratio between 4-chloro and 2-chlorodimethoxybenzene.

\section{Conclusions}

In summary, we have shown that under visible light irradiation K-PHI catalyzes formation of "HOHal" species from $\mathrm{Hal}^{-}$and $\mathrm{O}_{2}$ in aqueous media. According to the mechanism of this reaction "HOHal" species can be generated along both paths of photoredox chemistry: 1) quenching of $\mathrm{Hal}^{+}$, the product of $\mathrm{Hal}^{-}$two electron oxidation, by water and 2) reaction between $\mathrm{H}_{2} \mathrm{O}_{2}$, the product of $\mathrm{O}_{2}$ reduction, with $\mathrm{HCl}$. The generated electrophilic "HOCl" species was employed in oxidative halogenation of electron rich aromatic compounds. A solution of $\mathrm{NaCl}$ in water, seawater mimic, was also demonstrated to serve as a chlorinating agent. Potentially this reaction can also be expanded to run polyhalogenation of aromatic compounds, if water is replaced by a more oxidation stable, but ion solvating medium, e.g. ionic liquids. Also we have showed that the proposed approach is also applicable for bromination of aromatic compounds.

\section{Acknowledgements}


The authors are grateful to the Deutsche Forschungsgemeinschaft for the financial support (DFGAn 156 13-1). MA thanks the DFG Excellence Cluster "Unicat" for discussions and general support.

\section{Appendix A. Supplementary data}

\section{References}

[1] A. Podgoršek, M. Zupan, J. Iskra, Oxidative Halogenation with "Green" Oxidants: Oxygen and Hydrogen Peroxide, Angew. Chem. Int. Ed., 48 (2009) 84248450.

[2] T. Hering, B. Mühldorf, R. Wolf, B. König, Halogenase-Inspired Oxidative Chlorination Using Flavin Photocatalysis, Angew. Chem. Int. Ed., 55 (2016) $5342-$ 5345 .

[3] L. Zhang, X. Hu, Room temperature C(sp2)-H oxidative chlorination via photoredox catalysis, Chem. Sci., 8 (2017) 7009-7013.

[4] S. Liu, Q. Zhang, H. Li, Y. Yang, X. Tian, A. Whiting, A Visible - Light - Induced $\alpha$ - H Chlorination of Alkylarenes with Inorganic Chloride under NanoAg@AgCl, Chemistry - A European Journal, 21 (2015) 9671-9675.

[5] S. Liu, Q. Zhang, X. Tian, S. Fan, J. Huang, A. Whiting, Highly selective halogenation of unactivated $\mathrm{C}\left(\mathrm{sp}^{3}\right)-\mathrm{H}$ with $\mathrm{NaX}$ under co-catalysis of visible light and Ag@AgX, Green Chem., 20 (2018) 4729-4737.

[6] T. Song, B. Zhou, G.-W. Peng, Q.-B. Zhang, L.-Z. Wu, Q. Liu, Y. Wang, Aerobic Oxidative Coupling of Resveratrol and its Analogues by Visible Light Using Mesoporous Graphitic Carbon Nitride (mpg- $\mathrm{C}_{3} \mathrm{~N}_{4}$ ) as a Bioinspired Catalyst, Chem. Eur. J., 20 (2014) 678-682.

[7] Y. Chen, J. Zhang, M. Zhang, X. Wang, Molecular and textural engineering of conjugated carbon nitride catalysts for selective oxidation of alcohols with visible light Chem. Sci., 4 (2013) 3244-3248.

[8] P. Zhang, Y. Wang, H. Li, M. Antonietti, Metal-free oxidation of sulfides by carbon nitride with visible light illumination at room temperature, Green Chem., 14 (2012) 1904-1908

[9] Y. Zhao, M. Shalom, M. Antonietti, Visible light-driven graphitic carbon nitride $\left(g-C_{3} N_{4}\right)$ photocatalyzed ketalization reaction in methanol with methylviologen as efficient electron mediator, Appl. Catal., B, 207 (2017) 311-315.

[10] L. Li, D. Cruz, A. Savateev, G. Zhang, M. Antonietti, Y. Zhao, Photocatalytic cyanation of carbon nitride scaffolds: Tuning band structure and enhancing the performance in green light driven C-S bond formation, Appl. Catal., B, 229 (2018) 249-253.

[11] A.U. Meyer, V.W.-h. Lau, B. König, B.V. Lotsch, Photocatalytic Oxidation of Sulfinates to Vinyl Sulfones with Cyanamide-Functionalised Carbon Nitride, Eur. J. Org. Chem., (2017) 2179-2185.

[12] Y. Zhao, M. Antonietti, Visible-Light-Irradiated Graphitic Carbon Nitride Photocatalyzed Diels-Alder Reactions with Dioxygen as Sustainable Mediator for Photoinduced Electrons, Angew. Chem. Int. Ed., 56 (2017) 9336-9340.

[13] G. Zhang, A. Savateev, Y. Zhao, L. Li, M. Antonietti, Advancing the $n \rightarrow \pi^{*}$ electron transition of carbon nitride nanotubes for $\mathrm{H}_{2}$ photosynthesis, J. Mater Chem. A, 5 (2017) 12723-12728.

[14] S. Cao, Q. Huang, B. Zhu, J. Yu, Trace-level phosphorus and sodium co-doping of g-C3N4 for enhanced photocatalytic H2 production, J. Power Sources, $351(2017)$ 151-159.

[15] Y. Zhu, A. Marianov, H. Xu, C. Lang, Y. Jiang, Bimetallic Ag-Cu Supported on Graphitic Carbon Nitride Nanotubes for Improved Visible-Light Photocatalytic Hydrogen Production, ACS Applied Materials \& Interfaces, 10 (2018) 9468-9477.

[16] Y. Zhu, T. Wan, X. Wen, D. Chu, Y. Jiang, Tunable Type I and II heterojunction of CoOx nanoparticles confined in g-C3N4 nanotubes for photocatalytic hydrogen production, Appl. Catal., B, 244 (2019) 814-822.

[17] S. Cao, Y. Li, B. Zhu, M. Jaroniec, J. Yu, Facet effect of Pd cocatalyst on photocatalytic CO2 reduction over g-C3N4, J. Catal., 349 (2017) $208-217$.

[18] A. Savateev, M. Antonietti, Heterogeneous Organocatalysis for Photoredox Chemistry, ACS Catal., 8 (2018) 9790-9808.

[19] A. Savateev, I. Ghosh, B. König, M. Antonietti, Photoredox Catalytic Organic Transformations using Heterogeneous Carbon Nitrides, Angew. Chem. Int. Ed., 57 (2018) 15936-15947.

[20] D. Dontsova, S. Pronkin, M. Wehle, Z. Chen, C. Fettkenhauer, G. Clavel, M. Antonietti, Triazoles: A New Class of Precursors for the Synthesis of Negatively Charged Carbon Nitride Derivatives, Chem. Mater., 27 (2015) 5170-5179.

[21] Z. Chen, A. Savateev, S. Pronkin, V. Papaefthimiou, C. Wolff, M.G. Willinger, E. Willinger, D. Neher, M. Antonietti, D. Dontsova, 'The easier the better' preparation of efficient photocatalysts - metastable poly(heptazine imide) salts, Adv. Mater., 29 (2017) 1700555.

[22] A. Savateev, S. Pronkin, M. Willinger, M. Antonietti, D. Dontsova, Towards organic zeolites and inclusion catalysts: heptazine imide salts can exchange metal cations in the solid state, Chem. - Asian J., 12 (2017) 1517-1522.

[23] A. Savateev, S. Pronkin, J.D. Epping, M. Willinger, C. Wolff, D. Neher, M. Antonietti, D. Dontsova, Potassium Poly(heptazine imides) from Aminotetrazoles: Shifting Band Gaps of Carbon Nitride-like Materials by $0.7 \mathrm{~V}$ for More Efficient Solar Hydrogen and Oxygen Evolution, ChemCatChem, (2017) 167-174.

[24] G.-h. Moon, M. Fujitsuka, S. Kim, T. Majima, X. Wang, W. Choi, Eco-Friendly Photochemical Production of $\mathrm{H}_{2} \mathrm{O}_{2}$ through $\mathrm{O}_{2}$ Reduction over Carbon Nitride Frameworks Incorporated with Multiple Heteroelements, ACS Catal., 7 (2017) 2886-2895.

[25] G. Zhang, G. Li, Z.-a. Lan, L. Lin, A. Savateev, T. Heil, S. Zafeiratos, X. Wang, M. Antonietti, Optimizing Optical Absorption, Exciton Dissociation, and Charge Transfer of a Polymeric Carbon Nitride with Ultrahigh Solar Hydrogen Production Activity, Angew. Chem. Int. Ed., 56 (2017) 13445-13449.

[26] M. Antonietti, A. Savateev, Splitting Water by Electrochemistry and Artificial Photosynthesis: Excellent Science but a Nightm are of Translation?, Chem. Rec., (2018).

[27] B. Kurpil, A. Savateev, V. Papaefthimiou, S. Zafeiratos, T. Heil, S. Özenler, D. Dontsova, M. Antonietti, Hexaazatriphenylene doped carbon nitridesBiomimetic photocatalyst with superior oxidation power, Appl. Catal., B, 217 (2017) 622-628.

[28] B. Kurpil, B. Kumru, M. Antonietti, A. Savateev, Carbon nitride creates thioamides in high yields by the photocatalytic Kindler reaction, Green Chem., 20 (2018) 838-842.

[29] B. Kurpil, M. Antonietti, A. Savateev, Photooxidation of $\mathrm{N}$-acylhydrazones to 1,3,4-Oxadiazoles Catalyzed by Heterogeneous Visible-Light-Active Carbon Nitride Semiconductor, Appl. Catal., B, 228 (2018) 97-102.

[30] A. Savateev, B. Kurpil, A. Mishchenko, G. Zhang, M. Antonietti, A "waiting" carbon nitride radical anion: a charge storage material and key intermediate in direct C-H thiolation of methylarenes using elemental sulfur as the "S"-source, Chem. Sci., 9 (2018) 3584-3591.

[31] B. Kurpil, Y. Markushyna, A. Savateev, Visible-Light-Driven Reductive (Cyclo)Dimerization of Chalcones over Heterogeneous Carbon Nitride Photocatalyst, ACS Catal., doi: 10.1021/acscatal.8b04182 (2019) 1531-1538.

[32] A. Weidinger, M. Waiblinger, B. Pietzak, T.A. Murphy, Atomic nitrogen in C60: N@C60. Appl. Phys. A, 66 (1998) 287-292.

[33] M. Aldissi, S.P. Armes, X-ray photoelectron spectroscopy study of bulk and colloidal polyaniline, Macromolecules, 25 (1992) $2963-2968$.

[34] N.A. Rodriguez, A. Savateev, M.A. Grela, D. Dontsova, Facile Synthesis of Potassium Poly(heptazine imide) (PHIK)/Ti-Based Metal-Organic Framework (MIL-125-NH2) Composites for Photocatalytic Applications, ACS Applied Materials \& Interfaces, 9 (2017) 22941-22949.

[35] A. Mohammad, H.A. Liebhafsky, The Kinetics of the Reduction of Hydrogen Peroxide by the Halides, J. Am. Chem. Soc., 56 (1934) 1680-1685.

[36] S.T. Martin, H. Herrmannt, M.R. Hoffmann, Time-resolved Microwave Conductivity. Part 2.-Quantum-sized TiO, and the Effect of Adsorbates and Light Intensity on Charge-carrier Dynamics, J. Chem. Soc., Faraday Trans., 90 (1994) 3323-3330. 